Arq. Bras. Med. Vet. Zootec., v.68, n.6, p.1453-1457, 2016

\title{
Implante de Port-o-Cath para quimioterapia antineoplásica em um canino: relato de caso
}

\author{
[Implant of Port-o-Cath for antineoplastic chemotherapy \\ in a canine: case report] \\ E.R. Santos ${ }^{1}$, N.S. Rosa ${ }^{1}$, B.S. Barni ${ }^{1}$, M.P. Oliveira ${ }^{1}$, J.S. Venâncio ${ }^{1}$, E.A. Contesini ${ }^{2}$, \\ M.S. Muccillo ${ }^{3}$, D. Driemeier ${ }^{2}$ \\ ${ }^{1}$ Residente - Hospital de Clínicas Veterinárias - Universidade Federal do Rio Grande do Sul - Porto Alegre, RS \\ ${ }^{2}$ Faculdade de Veterinária - Universidade Federal do Rio Grande do Sul - Porto Alegre, RS \\ ${ }^{3}$ Hospital de Clínicas Veterinárias - Universidade Federal do Rio Grande do Sul - Porto Alegre, RS
}

\section{RESUMO}

O Port-o-Cath é um cateter venoso central totalmente implantável, o qual permite a infusão de quimioterápicos, hemoderivados, nutrição parenteral e coleta de sangue. Este trabalho relata o caso de um canino com um tumor no membro pélvico que foi tratado cirurgicamente e recebeu o cateter para a quimioterapia antineoplásica. A paciente possuía um sarcoma sinovial, tumor incomum em cães, que acomete normalmente as articulações e exige terapia multimodal. $\mathrm{O}$ tratamento foi feito em duas etapas; na primeira, o membro foi amputado e, na segunda, foi realizado o implante do Port-o-Cath e exérese de nódulo metastático. A cadela respondeu satisfatoriamente à cirurgia e realizou as sessões de quimioterapia com perfeito funcionamento do cateter, indo a óbito por outras causas 90 dias após o procedimento. O implante de Port-o-Cath para a quimioterapia é um grande aliado à terapia multimodal preconizada para o câncer, podendo o cateter ser considerado em pacientes que necessitem de medicação quimioterápica por longo período.

Palavras-chave: cateter venoso central totalmente implantável, sarcoma de tecidos moles, oncologia

\begin{abstract}
The Port-a-Cath is a totally implantable central venous catheter that allows the infusion of chemotherapeutic agents, blood products, parenteral nutrition, and blood sample collection. This work reports the case of a dog with a tumor in the pelvic limb that was treated surgically and received the catheter for cancer chemotherapy. The patient was diagnosed with synovial sarcoma, an uncommon tumor in dogs that usually affects the joints and requires multimodal therapy. The treatment was done in two steps, in the first the limb was amputated and in the second the Port-a-Cath implantation and the excision of the metastatic nodule were performed. The dog responded well to surgery and chemotherapy sessions held with proper functioning of the catheter, and died from other causes 90 days after the procedure. The implantation of Port-a-Cath for chemotherapy is a great ally of multimodal therapy recommended for cancer, so this catheter may be considered for patients requiring chemotherapy medication for a long period.
\end{abstract}

Keywords: totally implantable central venous catheter, soft tissue sarcoma, oncology

\section{INTRODUÇÃO}

Cateteres venosos centrais (CVC) podem ser de curta ou longa permanência, semi-implantáveis ou totalmente implantáveis (Port-o-Cath). A

Recebido em 27 de setembro de 2015

Aceito em 9 de junho de 2016

E-mail: eduardo.rosasantos@gmail.com primeira utilização de um cateter venoso central totalmente implantável (CVC-TI) em humanos data de 1970, com maior difusão na década seguinte. Em pessoas, são utilizados devido ao conforto possibilitado por evitar repetidas punções venosas em tratamentos quimioterápicos 
longos e por apresentar menor risco de complicações em relação aos cateteres de curta permanência (Ortolani et al., 2013). A quimioterapia sem o uso do Port-o-Cath requer várias punções que, somadas às características irritantes e vesicantes das medicações, levam à fragilidade e ao enrijecimento vascular, dificultando a venóclise e favorecendo o extravasamento, o que é perigoso no tratamento oncológico (Vasques et al., 2009).

O CVC-TI permite a infusão de quimioterápicos, hemoderivados, nutrição parenteral e coleta de sangue. Consiste em um cateter de silicone ou poliuretano e um port (câmara coberta por silicone puncionável) e é implantado cirurgicamente. $\mathrm{O}$ acesso é feito puncionando-se a pele sobre o port com agulha não cortante (Huber). As principais complicações são obstrução, infiltração, extravasamento e infecção (Vasques et al., 2009). Em cães, seu uso ainda é incipiente, mas ele pode ser colocado de forma semelhante ao CVC de curta permanência, utilizando-se normalmente o acesso jugular, ou ainda outros acessos menos frequentes, como a veia omobraquial (Radlinsky e Koenig, 2008). Sua implantação pode ser feita com auxílio de fluoroscopia, eletrocardiograma (ECG) ou guiada por ultrassom (US), e seu correto posicionamento pode ser avaliado com radiografia torácica ou ultrassonografia (Claude et al., 2010).

O sarcoma sinovial (SS) é um tumor maligno incomum de origem mesenquimal e tem sido reportado principalmente em cães de médiogrande porte, entre sete e oito anos de idade. Os locais mais acometidos são as articulações do joelho e do cotovelo, e as metástases ocorrem mais frequentemente em pulmões e linfonodos (França et al., 2004; Yamate et al., 2006). Para o tratamento, são indicadas a exérese local e a amputação do membro, associadas à quimioterapia e à radioterapia (Pinto et al., 2004; Gibbons et al., 2011).

Objetivou-se neste relato descrever um caso atendido no Hospital de Clínicas Veterinárias (HCV) da Universidade Federal do Rio Grande do Sul (UFRGS) de uma cadela submetida a um singular implante de Port-o-Cath para tratamento quimioterápico e seu acompanhamento em curto prazo.

\section{CASUÍSTICA}

Um canino, fêmea, sem raça definida, de aproximadamente 10 anos e pesando $28 \mathrm{~kg}$, foi atendido no HCV-UFRGS com grande aumento de volume no membro pélvico direito (MPD). $\mathrm{Na}$ anamnese, o responsável relatava que o tumor evoluía há dois meses com crescimento constante e se referia ainda à claudicação. No exame físico, o MPD apresentava tumoração de cerca de $25 \mathrm{x}$ $20 \mathrm{~cm}$ envolvendo o joelho e a maior parte do fêmur e tíbia, de consistência firme e aderida.

Suspeitando-se de neoplasia, foi realizada uma biópsia aspirativa por agulha fina (BAAF), e seu resultado foi sugestivo de tumor mesenquimal maligno. Radiografias torácicas não mostraram nódulos pulmonares metastáticos e alterações na silhueta cardíaca. Ecografia não apontou qualquer alteração relevante. Ecocardiograma indicou insuficiência mitral e tricúspide discretas. Eritrograma mostrou anemia normocítica hipocrômica (hematócrito 32\%), enquanto leucograma e contagem de plaquetas e estavam dentro da normalidade. No perfil bioquímico, a albumina, a creatinina e a alanina aminotransferase estavam normais, e a fosfatase alcalina elevada (305UI/L - referência: < 156UI/L). Baseando-se na BAAF e no histórico, foi indicada a amputação do membro. A cirurgia foi realizada de forma rotineira. No momento da revisão pós-operatória, cerca de 10 dias depois, notou-se um nódulo subcutâneo na região cervical dorsal de aproximadamente $9 \times 6 \mathrm{~cm}$, no qual foi realizada BAAF, que sugeriu ser novamente tumor mesenquimal maligno de origem indeterminada. Dessa forma, foi indicada a exérese do nódulo cervical e o implante de CVC-TI para quimioterapia antineoplásica.

No segundo procedimento, inicialmente foi realizada a exérese do nódulo metastático. Em seguida, o lado direito do pescoço, previamente tricotomizado, foi preparado assepticamente para a colocação do CVC-TI pela técnica percutânea. A jugular foi puncionada no seu terço médio, e pela agulha foi inserido um fio-guia. Logo após, a agulha foi retirada, o orifício dilatado, e o fioguia retirado para a progressão do cateter. $\mathrm{Na}$ sequência, o terço dorsal do pescoço foi incisado e preparado para a colocação do port, cerca de $15 \mathrm{~cm}$ de distância do local de inserção do cateter. Entre os dois foi feito um túnel subcutâneo para a passagem do cateter e conexão 
com o port, sendo este suturado em toda circunferência com náilon 2-0 em pontos isolados simples (PIS). Após a conexão, o sistema foi testado e o funcionamento mostrou-se adequado. A dermorrafia foi realizada com náilon 2-0 em PIS (Fig. 1). Realizou-se radiografia para certificação do posicionamento do cateter, constatando-se neste momento que ele avançou pelo átrio direito, ficando erroneamente posicionado na cava caudal, entretanto isto não causou prejuízo ao seu uso ou à saúde do animal (Fig. 2).

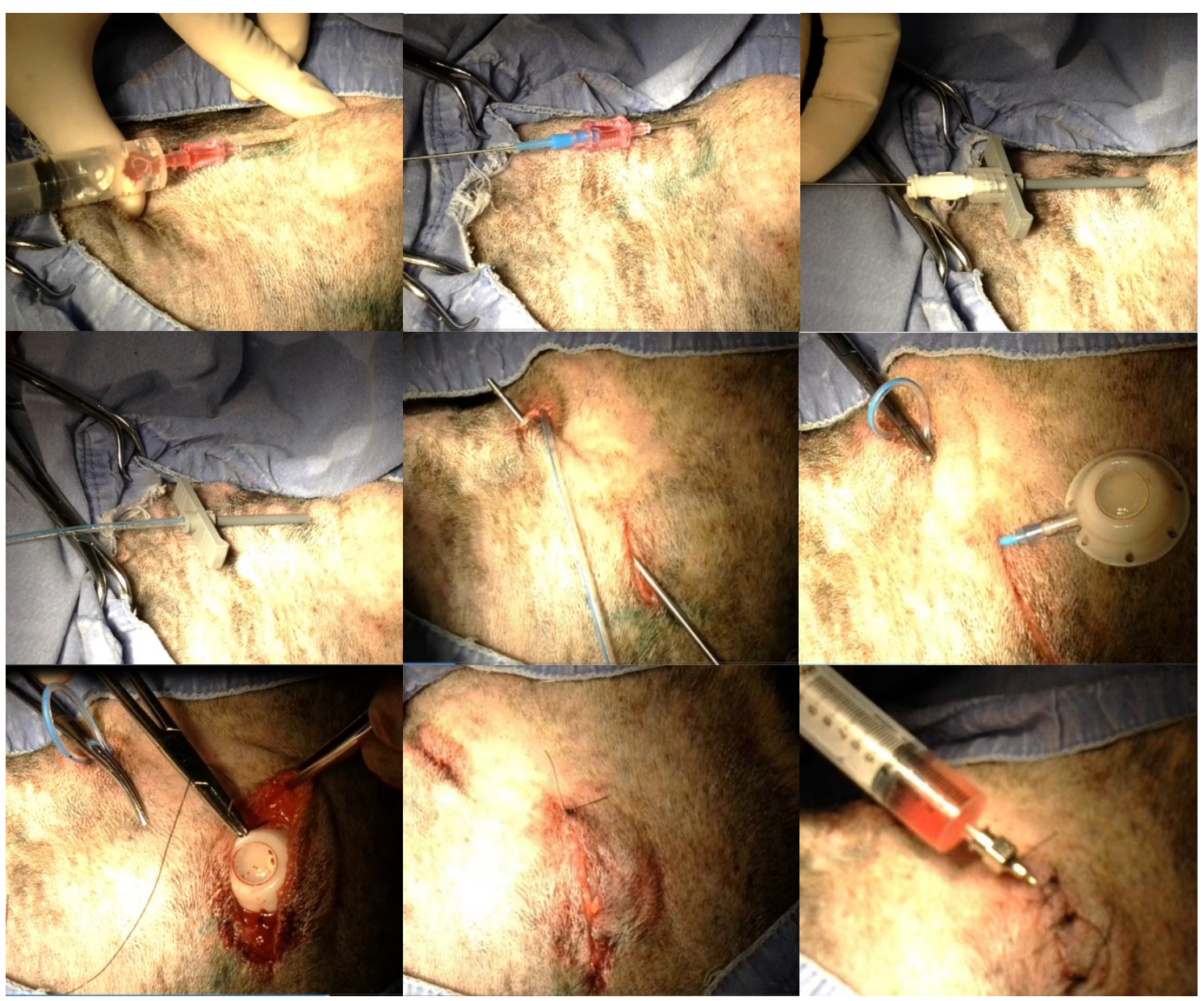

Figura 1. Etapas da colocação do CVC-TI. 1- Punção da jugular. 2- Introdução do fio-guia. 3- Inserção do dilatador. 4- Progressão do cateter. 5- Tunelização subcutânea. 6- Passagem do cateter pelo túnel e conexão com o port. 7- Início da sutura do port. 8- Aspecto ao primeiro ponto de pele. 9- Teste de funcionamento final. (Créditos: M.S. Muccillo).

$\mathrm{Na}$ revisão, seis dias após, o exame físico não mostrou qualquer parâmetro alterado, a cicatrização estava adequada, os pontos de pele foram retirados e, em novo hemograma e perfil bioquímico, todos os parâmetros estavam normais. Os histopatológicos de ambos os nódulos tiveram como diagnóstico sarcoma sinovial, e a paciente retornou ao hospital em nove dias para aplicação quimioterápica. Para a quimioterapia, a pele sobre o port foi higienizada com álcool e clorexidine $2 \%$; em seguida, o port foi puncionado com agulha de Huber e foi injetado $\mathrm{NaCl}$ 0,9\% sem resistência. Logo após, foram realizados aspiração e teste de refluxo de sangue e, então, o acoplamento do quimioterápico ao sistema e sua administração (Fig. 3). Ao final, foi infundido $\mathrm{NaCl} 0,9 \%$, e a paciente foi liberada. 


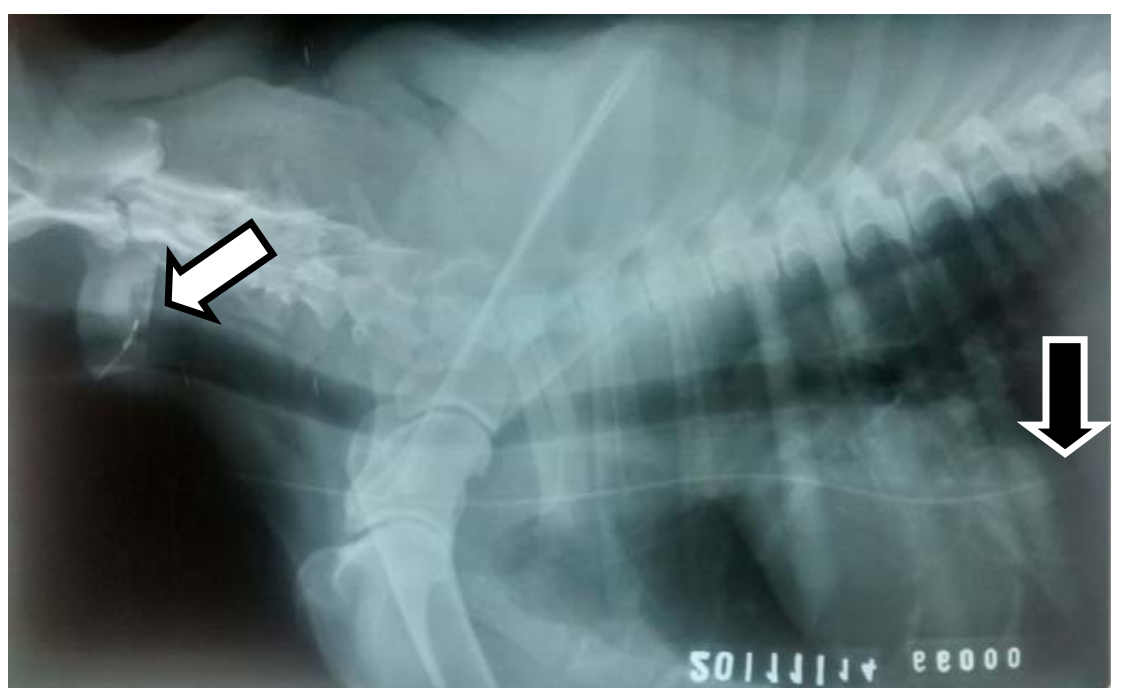

Figura 2. Radiografia torácica laterolateral mostrando o trajeto do cateter, o port (seta branca) e a extremidade do cateter (seta preta) na cava caudal.

A paciente retornou para mais três sessões quimioterápicas com intervalo de 21 dias cada. Ao $89^{\circ}$ dia de implante, ela retornou ao hospital com diversas alterações clínicas, indo a óbito no $90^{\circ}$ dia sem diagnóstico conclusivo. Esclareceuse, por meio da necropsia, que a aprovável causa mortis foi feocromocitoma adrenal com metástase em rins, pulmões e linfonodos.

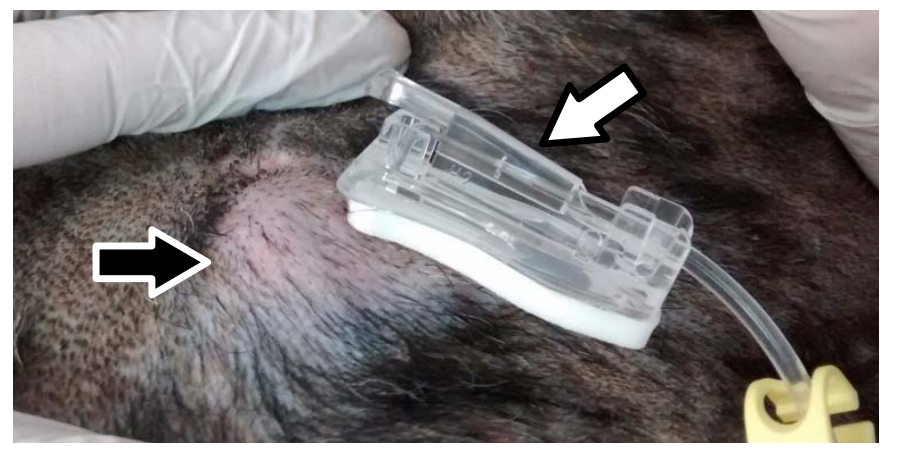

Figura 3. Punção do port (seta preta) para a primeira sessão de quimioterapia, utilizando-se agulha de Huber com sistema de segurança (seta branca).

\section{DISCUSSÃO}

O implante do Port-o-Cath propriamente dito é semelhante ao CVC de curta permanência, e o contraponto pode ser feito com base nesses estudos, devido à escassez de trabalhos abordando o CVC-TI. A implantação foi realizada pela técnica percutânea por punção jugular e progressão às cegas, ou seja, sem auxílio de ECG, US ou fluoroscópio. No estudo de Claude et al. (2010), foram comparadas duas técnicas de colocação (ECG-guiada e às cegas), e a probabilidade de se colocar o dispositivo corretamente utilizando-se qualquer uma delas foi a mesma, embora os estudos humanos apontem maior confiabilidade na técnica ECGguiada.

Uma alternativa à jugular é a inserção na veia omobraquial, utilizada no trabalho de Radlinsk e Koenig (2008) para colocação de CVC, devido à impossibilidade de uso das jugulares, porém esta necessita de maior experiência e possui restrição de tamanho do paciente. Apesar disso, o dispositivo (ao menos o CVC de curta permanência) pode ser implantado nesse sítio com segurança, conforto ao paciente e facilidade de uso. No trabalho de Portillo et al. (2006), foram comparadas duas técnicas de colocação de CVC por estudantes de veterinária. Cerca de 
$70 \%$ dos alunos conseguiram posicionar corretamente o cateter na primeira tentativa, mostrando que a técnica não apresenta grande dificuldade e a curva de aprendizado é curta. Em um dos estudos mais completos sobre o tema, Valentini et al. (2012) implantaram 12 CVC-TI em cães para quimioterapia antineoplásica ao longo de dois anos e meio. Durante o uso, foi notada pelos autores uma aflição ou angústia nos pacientes durante a perfuração da pele/port para a quimioterapia, o que não aconteceu no caso aqui relatado, já que a paciente permaneceu bastante tranquila durante o procedimento.

O único inconveniente encontrado na colocação do cateter foi o seu avanço excessivo, levando à localização errônea na veia cava caudal. De acordo com autores consultados por Claude et al. (2010), isto pode levar a tamponamento cardíaco, arritmias, hemotórax, flebite, perfuração e sepse, contudo essas complicações não foram observadas na paciente durante a permanência e o uso do cateter. Dos 12 cães do estudo de Valentini et al. (2012), um terço teve o cateter removido devido a complicações (infecção/fístulas) e, como neste caso, dois terços permaneceram com o dispositivo até o óbito.

No trabalho de Ortolani et al. (2013), 13,1\% dos CVC-TI implantados em crianças e adolescentes apresentaram problemas. Em média, os cateteres permaneceram nos pacientes $506,3 \pm 363,7$ dias com o maior tempo de permanência de 1335 dias; em geral, $11 \%$ dos pacientes permaneceram com o Port-o-Cath por mais de 900 dias. Neste caso, com 90 dias após o implante e tendo passado por quatro sessões quimioterápicas, o cateter seguia com funcionamento adequado e a paciente não apresentava sinais de infecção ou rejeição, contudo esta acabou indo a óbito devido a um feocromocitoma metastático no $90^{\circ}$ dia pósimplante.

\section{CONCLUSÃO}

O implante de Port-o-Cath para a quimioterapia antineoplásica é grande aliado à terapia multimodal preconizada para o tratamento do câncer, uma vez que atua na terapêutica e na qualidade de vida do paciente. O implante de
CVC-TI pode ser considerado em pacientes que necessitem de quimioterapia por longo período, contudo são necessários mais trabalhos em pacientes veterinários para avaliar os riscos e as complicações e difundir o seu uso.

\section{REFERÊNCIAS}

CLAUDE, A.K.; RIEDESEL, D.H.; RIEDESEL, E.A. Electrocardiography-guided and retrospective analysis of central venous catheter placement in the dog. Vet. Anaesth. Analg., v.37, p.97-105, 2010.

FRANÇA, S.A.; SERAKIDES, R.; SILVA, A.E. et al. Sarcoma sinovial extra-articular em cão. Arq. Bras. Med. Vet. Zootec., v.56, p.683-686, 2004.

GIBBONS, D.S.; BENNET, A.D.; TREUIL, P.L. Palliative radiation therapy in the treatment of canine appendicular synovial sarcoma. J. Am. Anim. Hosp. Assoc., v.47, p.359-364, 2011.

ORTOLANI, L.; GASPARINO, R.C.; TRALDI, M.C. Complicações associadas ao uso de cateter totalmente implantável em crianças e adolescentes. Rev. Bras. Cancerol., v.59, p.51-56, 2013.

PINTO, A.C.B.C.F.; TATARUNAS, A.C.; CHALITA, M.C.C. et al. Sarcoma sinovial da articulação antebraquiocárpica em cão. Rev. Educ. Contin. CRMV-SP, v.7, p.34-39, 2004.

PORTILLO, E.; MACKIN, A.; HENDRIX, P.K. et al. Comparison of themodified Seldinger and throughtheneedle jugular catheter placement techniques in the dog. J. Vet. Emerg. Crit. Care, v.16, p.88-95, 2006.

RADLINSKY, M.G.; KOENIG, A. Central venous access to the cranial vena cava via the omobrachial vein in the dog. J. Vet. Emerg. Crit. Care, v.18, p.659$662,2008$.

VALENTINI, F.; FASSONE, F.; POZZEBON, A. et al. Use of totally implantable vascular access port with mini-invasive Seldinger technique in 12 dogs undergoing chemotherapy. Res. Vet. Sci., v.94, p.152157, 2012.

VASQUES, C.I.; REIS, P.E.D.; CARVALHO, E.C. Manejo do cateter venoso central totalmente implantado em pacientes oncológicos: revisão integrativa. Acta Paulista Enferm., v.22, p.696-701, 2009.

YAMATE, J.; AOTA, M.; KUWAMURA, M. et al. Synovial sarcoma of the tendon sheath in a dog. J. Vet. Med. Sci., v.68, p.983-986, 2006. 